Tropical Journal of Pharmaceutical Research January 2020; 19 (1): 169-181

ISSN: $1596-5996$ (print); 1596-9827 (electronic)

(c) Pharmacotherapy Group, Faculty of Pharmacy, University of Benin, Benin City, 300001 Nigeria.

\title{
In-silico design of novel 4-aminoquinolinyl analogs as potential anti-malaria agents using quantitative structure- activity relationships and ADMET approach
}

\author{
Bo $\mathrm{Wu}^{1}$, Jiao-Long Wang ${ }^{2}$, Liang $\mathrm{Li}^{3}$, Chun-Jie $\mathrm{Wu}^{2}$, Chong-Bo Zhao ${ }^{1,4 \star}$ \\ ${ }^{1}$ Department of Pharmaceutics, College of Pharmacy, Shaanxi University of Chinese Medicine, Xianyang, Shaanxi, ${ }^{2}$ School of \\ Pharmacy, Chengdu University of Traditional Chinese Medicine, Chengdu, 611137, ${ }^{3}$ Hospital of Chengdu University of \\ Traditional Chinese Medicine, Chengdu 610075, ${ }^{4}$ Department of Pharmaceutics, College of Pharmacy, Engineering \\ Technology Research Center of Shaanxi Administration of Chinese Herbal Pieces, Shaanxi University of Chinese Medicine, \\ Xianyang, Shaanxi, PR China
}

*For correspondence: Email: zhao_chongbo@126.com; Tel/Fax: +86-29-38185072

Sent for review: 23 August 2019

Revised accepted: 31 December 2019

\begin{abstract}
Purpose: To design and screen for potential anti-malaria agents based on a series of 4-aminoquinolinyl analogues.

Methods: Molecular fingerprint analysis was used for molecular partitioning of training and test sets. Acquired training sets were used for CoMFA and CoMSIA model construction after good alignment was achieved. Partial least squares analysis combined with external validation were used for model evaluation. Deep analysis of acquired contour maps was performed to summarize the substituent property requirements for further rational molecular design. Using the chosen models, activity prediction and subsequent ADMET investigation were performed to discover novel designed compounds with the desired properties.

Results: Three different set partitions for model establishment were obtained using fingerprint-based selection. Partition 02 offered an optimal CoMFA model $\left(r^{2}=0.964, q^{2}=0.605\right.$ and $\left.r^{2 p r e d}=0.6362\right)$ and the best CoMSIA model $\left(r^{2}=0.955, q^{2}=0.585\right.$ and $r^{2}$ pred $\left.=0.6403\right)$. Based on contour map analysis, a series of compounds were designed for activity prediction. Two of the compounds (wmx09, wmx25) were chosen for their ideal predicted biological activities. Subsequent ADMET investigation indicated that these compoundss have acceptable drug-like characteristics.

Conclusion: The screening reveals that compounds wmx09 and wmx25 have strong potential as antimalaria agents.
\end{abstract}

Keywords: Malaria, 4-Aminoquinolinyl, Molecular fingerprint, QSAR, ADMET

This is an Open Access article that uses a fund-ing model which does not charge readers or their institutions for access and distributed under the terms of the Creative Commons Attribution License (http://creativecommons.org/licenses/by/4.0) and the Budapest Open Access Initiative (http://www.budapestopenaccessinitiative.org/read), which permit unrestricted use, distribution, and reproduction in any medium, provided the original work is properly credited.

Tropical Journal of Pharmaceutical Research is indexed by Science Citation Index (SciSearch), Scopus, International Pharmaceutical Abstract, Chemical Abstracts, Embase, Index Copernicus, EBSCO, African Index Medicus, JournalSeek, Journal Citation Reports/Science Edition, Directory of Open Access Journals (DOAJ), African Journal Online, Bioline International, Open-J-Gate and Pharmacy Abstracts

\section{INTRODUCTION}

Malaria is widely distributed from latitude 60 degrees north to 30 degrees south. It is generally recognized as a fatal parasitic disease threat. Three billion two hundred million people in more than 90 countries of Africa, Southeast Asia, South Asia, Arabian peninsula, Central and South America. Malaria kills approximately 
400,000 people each year and children under the age of 5 years are a significant proportion of these deaths [1]. Plasmodium falciparum and Plasmodium vivax are associated with most malaria epidemics worldwide. However, most infections are caused by Plasmodium falciparum, which is responsible for more than $95 \%$ of reported malaria-related cases [2].

Due to fact that progress on malaria vaccine development has been insufficient, chemotherapy is the only option for malaria treatment [3]. Due to its efficacy, safety and drugaccessibility, chloroquine has been the most widely used malaria therapy since its firstly clinical application in 1944 [4]. However, the increasingly serious problem of chloroquine resistance has gradually become a primary reason for failures of malaria prevention and control. Hence, studies of structural modifications based on chloroquine analogs have found these anti-malarial candidates to have considerable scientific value, and they have received significant research attention in recent years. $[5,6]$.

Chemoinformatics-based computational approaches (e.g., molecular docking, dynamics, and quantitative structure-activity relationships [QSARs]) have resulted in numerous successful examples of novel candidate drug discovery $[7,8]$. Using appropriate statistical methods, quantitative structure-activity relationship analysis has been found to be an effective approach to generate physicochemical, structural, steric and electrostatic information for rational molecular design based on a series of analogs. Widely used during the last two decades, three-dimensional QSAR study analyzes relationship between structural features of compounds and their target properties in three-dimensional coordinates, Using this approach, researchers obtain visual interaction contour images and predict outcomes $[9,10]$.

In the present study, we performed a carefully designed QSAR study based on a series of 4aminoquinolinyl analogs. We aimed to discover potential anti-Plasmodium falciparum agents and new candidates for further molecular design for malaria therapy based on chloroquine substructure.

\section{EXPERIMENTAL}

\section{Datasets}

A totally of 48 different 4 -aminoquinolinyl analogs were derived during previous studies performed by the Prem M. S. Chauhan research team [1113]. To simplify the data format, all reported biological activity ( $\mathrm{IC}_{50}$ values) were translated to a negative logarithmic format $\left(\mathrm{plC}_{50}=-\lg \mid \mathrm{C}_{50}\right)$ and added into an attribute spreadsheet (Table 1 - Table 4).

\section{Molecule preparation}

All molecules were carefully sketched using software of ChemDraw Professional 15.0 (CambridgeSoft Corporation, USA; www.cambridgesoft.com). Each molecule was recorded as isolated model definition language (MDL) Molfile files. Discovery Studio 4.5 software (Biovea Inc, USA: www.biovea.com.) was used to generate molecular three-dimensional structures. The "Minimize ligands" protocol in Discovery Studio 4.5 was used for molecule minimization. The "Smart Minimizer" calculation algorithm was used to perform 1,000 steps of steepest descent with a root mean square (RMS) gradient tolerance of 3 , followed by conjugate gradient minimization [14]. The "Max steps" was set at 2,000, the "RMS Gradient" was set at $0.001 \mathrm{kcal} / \mathrm{mol} \AA$ and Merck Molecular Force Field was selected as input forcefield. All acquired molecular conformations were saved as Sybyl MOL2 files for further study.

\section{Clustering analysis}

To develop more robust QSAR models, a cluster analysis based on molecular fingerprint was performed [15]. Fingerprint of "MDL public keys" was used as the calculation precept to divide all 48 molecules into seven clusters [16]. One molecule was selected from each cluster for the test sets (i.e., seven molecules, or $15 \%$ of the total molecules). After a selection from each cluster based on a principle of sufficient structural diversity and gradient biological activity, three different test sets were built.

\section{QSAR studies}

\section{Molecule alignment}

All minimized molecular conformations were delivered to software of Sybyl X-2.1 (Tripos Inc. USA) for CoMFA and CoMSIA QSAR studies. Following the cluster analysis results, three training-test molecule divisions were manually performed and saved as Sybyl databases. Due to its best reported biological activity, molecule 35 was selected as reference for molecular alignment. Each set was aligned using Sybyl "Align Database" function following maximum common substructure method [17]. 
Wu et al

Table 1: 4-Aminoquinolinyl analogs with reported activities (Comp01 - Comp13)<smiles>[R]Nc1ccc(Nc2ccnc3cc(Cl)ccc23)cc1</smiles>

\begin{tabular}{|c|c|c|c|}
\hline Comp. & $\mathbf{R}$ & $\mathrm{IC}_{50}(\mathrm{nM})$ & $\mathrm{plC}_{50}$ \\
\hline 01 & & 38.77 & 7.412 \\
\hline 02 & & 23.13 & 7.636 \\
\hline 03 & & 18.53 & 7.732 \\
\hline 04 & & 38.34 & 7.416 \\
\hline 05 & & 37.07 & 7.431 \\
\hline 06 & & 40.88 & 7.388 \\
\hline 07 & & 19.69 & 7.706 \\
\hline 08 & & 291.06 & 6.536 \\
\hline 09 & & 43.94 & 7.357 \\
\hline 10 & & 20.78 & 7.682 \\
\hline 11 & & 25.38 & 7.596 \\
\hline 12 & & 91.75 & 7.037 \\
\hline 13 & & 47.74 & 7.321 \\
\hline
\end{tabular}


Wu et al

Table 2: 4-aminoquinolinyl analogs with reported activities (Comp14 - Comp27)

\begin{tabular}{|c|c|c|c|}
\hline Comp. & $\mathbf{R}$ & $\mathrm{IC}_{50}(\mathrm{nM})$ & $\mathrm{plC}_{50}$ \\
\hline 14 & & 33.65 & 7.473 \\
\hline 15 & & 43.81 & 7.358 \\
\hline 16 & & 152.35 & 6.817 \\
\hline 17 & & 81.47 & 7.089 \\
\hline 18 & & 41.5 & 7.382 \\
\hline 19 & & 19.03 & 7.721 \\
\hline 20 & & 31.52 & 7.501 \\
\hline 21 & & 26.05 & 7.584 \\
\hline 22 & & 27.78 & 7.556 \\
\hline 23 & & 29.65 & 7.528 \\
\hline 24 & & 37.63 & 7.424 \\
\hline 25 & & 12.44 & 7.905 \\
\hline 26 & & 44.49 & 7.352 \\
\hline 27 & & 82.85 & 7.082 \\
\hline
\end{tabular}


Wu et al

Table 3: 4-aminoquinolinyl analogs with reported activities (Comp28 - Comp38)

\begin{tabular}{|c|c|c|c|}
\hline Comp. & $\mathbf{R}$ & $\mathrm{IC}_{50}(\mathrm{nM})$ & $\mathrm{plC}_{50}$ \\
\hline 28 & & 63.5 & 7.197 \\
\hline 29 & & 11.88 & 7.925 \\
\hline 30 & & 6.41 & 8.193 \\
\hline 31 & & 16.13 & 7.792 \\
\hline 32 & & 19.49 & 7.710 \\
\hline 33 & & 16.74 & 7.776 \\
\hline 34 & & 4.21 & 8.376 \\
\hline 35 & & 3.6 & 8.444 \\
\hline 36 & & 50.79 & 7.294 \\
\hline 37 & & 13.62 & 7.866 \\
\hline 38 & & 7.06 & 8.151 \\
\hline
\end{tabular}


Table 4: 4-aminoquinolinyl analogs with reported activities (Comp39 - Comp48)

\begin{tabular}{|c|c|c|c|}
\hline Comp. & $\mathbf{R}$ & $\mathrm{IC}_{50}(\mathrm{nM})$ & $\mathrm{plC}_{50}$ \\
\hline 39 & & 4.87 & 8.312 \\
\hline 40 & & 6.22 & 8.206 \\
\hline 41 & & 21.86 & 7.660 \\
\hline 42 & & 35.19 & 7.454 \\
\hline 43 & & 28.52 & 7.545 \\
\hline 44 & & 23.63 & 7.627 \\
\hline 45 & & 48.64 & 7.313 \\
\hline 46 & & 20.67 & 7.685 \\
\hline 47 & & 74.34 & 7.129 \\
\hline 48 & & 32.66 & 7.486 \\
\hline
\end{tabular}

\section{CoMFA and CoMSIA field calculation}

Sybyl was used to calculate CoMFA and CoMSIA fields for each aligned training set: An sp3 carbon atom with charge of +1 was launched to probe steric and electrostatic fields data, $4 \AA$ beyond every direction for each molecule was calculated and then a region file was created. When performing CoMSIA calculations, an accessional hydrophobicity property of +1 and a hydrogen bond property of +1 were added to the probe atom to calculate 
the hydrophobic, hydrogen bond acceptor and hydrogen bond donor fields energies. We used $30.0 \mathrm{kcal} / \mathrm{mol}$ as the steric and electrostatic cutoffs for the CoMFA field calculations and 0.3 as the attenuation factor for the CoMSIA field calculations [18]. The biological activity values were merged into the spreadsheets after they were recorded into a text document file.

\section{Partial least squares analysis}

Partial least squares regression analysis was performed to calculate correlation between molecular activities and created CoMFA and CoMSIA fields. The statistical analysis was performed according to a classical two-stage scheme. The first stage was a leave-one-out cross-validation analysis, which used the remaining model to predict one separated molecule. Based on the results of the leave-oneout analysis, a squared cross-validation coefficient $\left(\mathrm{q}^{2}\right)$ value and an optimum number of components $(\mathrm{N})$ were acquired. Using the optimum number of components value, the second stage of no validation analysis was performed. No validation analysis assisted us to acquire regression values for $r$ squared $\left(r^{2}\right)$, the standard error of estimate (SEE) and the $F$ values, which are important indicators for model evaluation. Based on the results for different training sets, every possible CoMFA and CoMSIA model was built and evaluated $[19,20]$.

\section{External validation analysis}

As prediction ability contributes majority of a QSAR model's validity, external validation must be performed [21]. Calculation of predictive $r^{2}$ $\left(r_{\text {pred }}^{2}\right)$ values was used as an indicator for model external validation:

$r_{\text {pred }}^{2}=($ SD-PRESS $) / S D$

Where SD was the sum of the squared deviations between the mean activities of the training set compounds and the reported activities of the test set compounds, and PRESS is the sum of squared deviations between reported and predicted activities of the test set compounds [22]. Test set molecules were delivered for external validation after alignment with molecule 35.

\section{Molecule design, applicability domain analysis, and activity prediction}

\section{Molecule design}

Contour maps are visual three-dimensional images created using QSAR models that display the interactions and correlations between molecular structural features with a certain field. Based on acquired contour maps, we performed a deep analysis to summarize the structural requirements for molecule design and acquire an in-house library consisting of a series of rationally designed compounds.

\section{Applicability domain analysis}

As the inherent "closed system" characteristic of every QSAR model limits its applicability, the applicability domains for created models should be calculated [23]. We performed optimum prediction space analysis to define the applicability domains for the models. We used the "optimum prediction space" function in the Discovery Studio software to automatically discriminate whether the designed molecules were located inside the applicability domains, based on Mahalanobis distance.

\section{Activity prediction}

Each designed molecule was optimized according to the method mentioned in Molecule Preparation section. Before prediction, each molecule was equally aligned using molecule 35 as the template. Molecules with better predicted biological activities were used for further study.

\section{ADMET prediction}

ADMET prediction studies were performed for screened molecules using "ADMET Descriptors" and "Toxicity Prediction" functions in Discovery Studio software. Comprehensive consideration of all these data was used to select more precise potential compounds [24,25].

\section{RESULTS}

\section{Clustering analysis}

The results for the cluster partition outcomes are presented in Table 5. We abided by the principle of sufficient structural diversity and gradient biological activity to carefully pick molecules for three different test sets.

\section{QSAR}

\section{Statistical data}

All molecules from the datasets were wellaligned when molecule 35 was used as a reference (Figure 1). Three overlapping training databases were then sent for statistical analysis. The evaluation criteria for the indicators were: 1 . high values of $r^{2}, q^{2}, r^{2}$ pred and $F ; 2$. low value for 
SEE; and 3. an optimum number of components staying within reasonable limits.

Table 5: Serial numbers for compounds from each cluster using model definition language public keys as analysis precepts

\begin{tabular}{|c|c|}
\hline $\begin{array}{l}\text { Cluster } \\
\text { number }\end{array}$ & Serial number of molecules \\
\hline 1 & $04,85,66,07,45$ \\
\hline 2 & 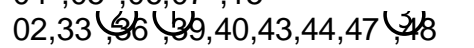 \\
\hline 3 & $14,18,19,22,23(3), 28 \bigcup 29,30<$ \\
\hline 4 & 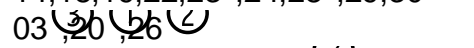 \\
\hline 5 & $\begin{array}{l}01,21,25,27,31,32,34,35,37,38, \\
41,42,45,46\end{array}$ \\
\hline $\begin{array}{l}6 \\
7\end{array}$ & 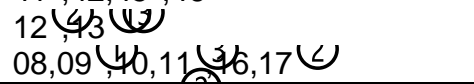 \\
\hline
\end{tabular}

Trolecules for test set 01; दrolecules for test set 02 ; 3 olecules for test set 03

For the CoMFA model selection, among the three candidates model 02 had most optimum outcomes $\left(r^{2}=0.964, q^{2}=0.605, F=153.831\right.$ and SEE $=0.078$ ), which indicated the robustness of the chosen model. External validation results also reinforced the selection of model 02: Model 02 was calculated to possess the best prediction ability in terms of the highest $r^{2}$ pred $=0.6362$. The optimum number of six components also met the "within reasonable limits" requirement. For CoMSIA model selection, model 02 was also the relative best statistical model.

Amongst all generated CoMSIA models, model CoMSIA EHA was selected not only for its best internal validation values $\left(r^{2}=0.955, q^{2}=0.585\right.$, $\mathrm{F}=184.54$ and SEE $=0.091)$, but also for its acceptable external validation value of $r_{\text {pred }}^{2}=$ 0.6403 and a reasonable optimum number of eight components (Table 6). Because model CoMSIA EHA consisted of the three electrostatic, hydrophobic, and hydrogen bond acceptor descriptor fields, it may also provide more information for further study. All molecules were aligned and predicted by both selected models. As presented in Table 7, Table 8, and Figure 2, the close proximities of predicted and reported activities proved the quality of selected models. Consequently, CoMFA and CoMSIA_EHA models generated using dataset 02 were chosen for further study.

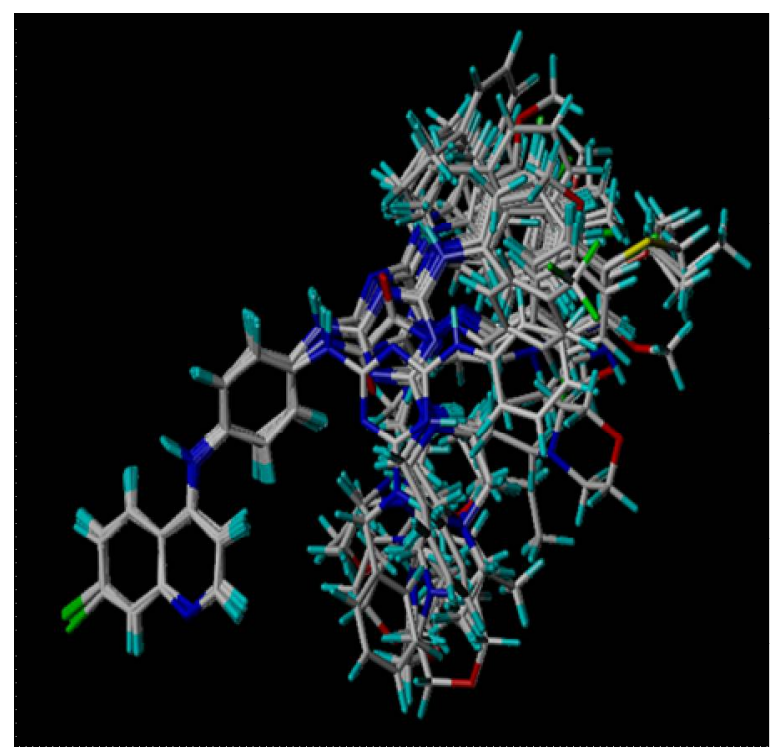

Figure 1: Molecular alignment based on the common substructure using molecule 35 as a reference

Table 6: Statistical values for evaluation of the quality of created CoMFA and CoMSIA models

\begin{tabular}{|c|c|c|c|c|c|c|}
\hline \multirow{2}{*}{ Model name } & \multicolumn{6}{|c|}{ Statistical parameter } \\
\hline & $n$ & $q^{2}$ & $r^{2}$ & SEE & $\boldsymbol{F}$ & $r_{\text {pred }}^{2}$ \\
\hline \multicolumn{7}{|l|}{ Training set 01} \\
\hline CoMFA & 4 & 0.461 & 0.840 & 0.135 & 87.599 & 0.4821 \\
\hline CoMSIA_SHA & 6 & 0.338 & 0.784 & 0.154 & 67.126 & 0.3241 \\
\hline COMSIA_SEA & 6 & 0.422 & 0.821 & 0.217 & 92.221 & 0.4195 \\
\hline $\begin{array}{l}\text { CoMSIA_SEHDA } \\
\text { Training set } 02\end{array}$ & 4 & 0.382 & 0.790 & 0.281 & \multicolumn{2}{|c|}{ Training set 02} \\
\hline CoMFA & 6 & 0.605 & 0.964 & 0.078 & 153.831 & 0.6362 \\
\hline COMSIA_EHA & 8 & 0.585 & 0.955 & 0.091 & 184.54 & 0.6403 \\
\hline COMSIA_SHA & 6 & 0.542 & 0.926 & 0.103 & 132.528 & 0.5185 \\
\hline COMSIA_SEHA & 8 & 0.522 & 0.912 & 0.155 & 143.851 & 0.5853 \\
\hline \multicolumn{7}{|l|}{ Training set 03} \\
\hline CoMFA & 6 & 0.558 & 0.924 & 0.101 & 133.423 & 0.5779 \\
\hline CoMSIA_SA & 4 & 0.509 & 0.826 & 0.150 & 85.675 & 0.5102 \\
\hline CoMSIA_EH & 9 & 0.533 & 0.901 & 0.089 & 112.980 & 0.5412 \\
\hline COMSIA EHA & 4 & 0.467 & 0.881 & 0.115 & 109.645 & 0.4732 \\
\hline
\end{tabular}


Table 7: Comparison of reported and predicted activities based on selected CoMFA and CoMSIA models (Comp01 - Comp24)

\begin{tabular}{|c|c|c|c|c|c|}
\hline Compound & Reported plC 50 & $\begin{array}{c}\text { CoMFA } \\
\text { Predicted plC }{ }_{50}\end{array}$ & Residual & $\begin{array}{c}\text { CoMSIA } \\
\text { Predicted pIC }\end{array}$ & Residual \\
\hline 01 & 7.412 & 7.429 & 0.017 & 7.423 & 0.011 \\
\hline 02 & 7.636 & 7.705 & 0.069 & 7.644 & 0.008 \\
\hline 03 & 7.732 & 7.653 & -0.079 & 7.626 & -0.106 \\
\hline 04 & 7.416 & 7.426 & 0.010 & 7.429 & 0.013 \\
\hline 05 & 7.431 & 7.465 & 0.034 & 7.445 & 0.014 \\
\hline 06 & 7.388 & 7.407 & 0.019 & 7.460 & 0.072 \\
\hline 07 & 7.706 & 7.480 & -0.226 & 7.553 & -0.153 \\
\hline 08 & 6.536 & 6.520 & -0.016 & 6.563 & 0.027 \\
\hline 09 & 7.357 & 7.335 & -0.022 & 7.339 & -0.018 \\
\hline 10 & 7.682 & 7.627 & -0.055 & 7.597 & -0.085 \\
\hline 11 & 7.596 & 7.580 & -0.016 & 7.576 & -0.020 \\
\hline 12 & 7.037 & 7.383 & 0.346 & 7.386 & 0.349 \\
\hline 13 & 7.321 & 7.344 & 0.023 & 7.336 & 0.015 \\
\hline 14 & 7.473 & 7.466 & -0.007 & 7.403 & -0.070 \\
\hline 15 & 7.358 & 7.385 & 0.027 & 7.402 & 0.044 \\
\hline 16 & 6.817 & 6.788 & -0.029 & 6.773 & -0.044 \\
\hline 17 & 7.089 & 6.766 & -0.323 & 6.674 & -0.115 \\
\hline 18 & 7.382 & 7.582 & 0.200 & 7.645 & 0.263 \\
\hline 19 & 7.721 & 7.586 & -0.135 & 7.652 & -0.069 \\
\hline 20 & 7.501 & 7.526 & 0.025 & 7.668 & 0.167 \\
\hline 21 & 7.584 & 7.611 & 0.027 & 7.580 & -0.004 \\
\hline 22 & 7.556 & 7.524 & -0.032 & 7.435 & -0.121 \\
\hline 23 & 7.528 & 7.509 & -0.019 & 7.457 & -0.071 \\
\hline 24 & 7.424 & 7.504 & 0.080 & 7.506 & 0.082 \\
\hline
\end{tabular}

Table 8: Comparison of reported and predicted activities based on selected CoMFA and CoMSIA models (Comp25 - Comp48).

\begin{tabular}{|c|c|c|c|c|c|}
\hline Comp. & Reported plC 50 & $\begin{array}{c}\text { CoMFA } \\
\text { Predicted plC }\end{array}$ & Residual & $\begin{array}{c}\text { CoMSIA } \\
\text { Predicted pIC }\end{array}$ & Residual \\
\hline 25 & 7.905 & 7.868 & -0.037 & 7.857 & -0.048 \\
\hline 26 & 7.352 & 7.648 & 0.296 & 7.766 & 0.314 \\
\hline 27 & 7.082 & 7.057 & -0.025 & 6.970 & -0.112 \\
\hline 28 & 7.197 & 7.294 & 0.097 & 7.333 & 0.136 \\
\hline 29 & 7.925 & 7.703 & -0.222 & 7.783 & -0.142 \\
\hline 30 & 8.193 & 8.076 & -0.117 & 7.826 & -0.267 \\
\hline 31 & 7.792 & 7.808 & 0.016 & 7.840 & 0.048 \\
\hline 32 & 7.710 & 7.822 & 0.112 & 7.797 & 0.087 \\
\hline 33 & 7.776 & 7.709 & -0.067 & 7.608 & -0.168 \\
\hline 34 & 8.376 & 8.424 & 0.048 & 8.391 & 0.015 \\
\hline 35 & 8.444 & 8.379 & -0.065 & 8.371 & -0.073 \\
\hline 36 & 7.294 & 7.291 & -0.003 & 7.293 & -0.001 \\
\hline 37 & 7.866 & 7.818 & -0.048 & 7.867 & 0.001 \\
\hline 38 & 8.151 & 8.121 & -0.030 & 8.064 & -0.087 \\
\hline 39 & 8.312 & 8.343 & 0.031 & 8.358 & 0.046 \\
\hline 40 & 8.206 & 8.201 & -0.005 & 8.185 & -0.021 \\
\hline 41 & 7.660 & 7.512 & -0.148 & 7.580 & -0.080 \\
\hline 42 & 7.454 & 7.502 & 0.048 & 7.569 & 0.115 \\
\hline 43 & 7.545 & 7.496 & -0.049 & 7.566 & 0.021 \\
\hline 44 & 7.627 & 7.640 & 0.013 & 7.621 & -0.006 \\
\hline 45 & 7.313 & 7.435 & 0.122 & 7.699 & 0.186 \\
\hline 46 & 7.685 & 7.765 & 0.080 & 7.664 & -0.021 \\
\hline 47 & 7.129 & 7.139 & 0.010 & 7.116 & -0.013 \\
\hline 48 & 7.486 & 7.530 & 0.044 & 7.516 & 0.030 \\
\hline
\end{tabular}



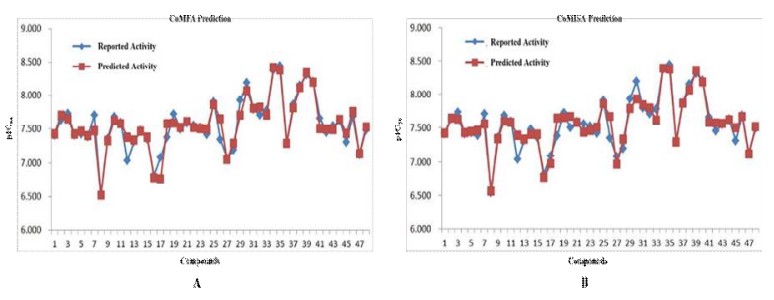

Figure 2: Scatter plots of reported activities versus predicted activities for all molecules based on created CoMFA and CoMSIA models.

\section{Contour map analysis}

To implement rational molecular designs, we performed a deep analysis focusing on the acquired contour maps. Overlap of molecule 35 with each contour map using three-dimensional coordinate to display the relationships between the most active compound and each target property. Figure $3 \mathrm{~A}$ presents the overlap figure for electrostatic contour map: Blue regions (positive electrostatic favored), located around nitrogen atom on arylamine group, indicated nitrogen atom is required at this position. Several red modules (negative electrostatic favored) associated with substituent groups at position two and position three on the aromatic ring. If electron-withdrawing groups are placed at these positions, this change may enhance the molecular activity. The terminal of aliphatic chain of the 4 - ethylpiperazine group was also associated with red modules. This result suggested that electron-withdrawing groups are required at these positions.

The overlapping figure for the steric contour map is presented in Figure 3B. Position two on the aromatic ring of the anilino group is sieged by yellow regions (negative steric favored) while green (positive steric favored) modules were located near position three. This result suggested that any substitutions with bulky properties should occur at position three. The piperazine group was conglutinated with green regions, which indicated that placement of a hexatomic ring at this site is a rational decision.

Figure $3 \mathrm{C}$ depicts the results for hydrophobic contour map: Hydrophilic favored regions (white regions) covered the arylamine group. This result indicated that increasing the hydrophilic properties of substituent groups is beneficial at this location. Figure 3D presents the results for hydrogen bond acceptor contour map: It suggests that groups with hydrogen bonding ability can be added to the piperazine ring because a large purple module (hydrogen bond acceptor favored) was located nearby. The aliphatic chain terminal also possesses the ability to form hydrogen bonds.

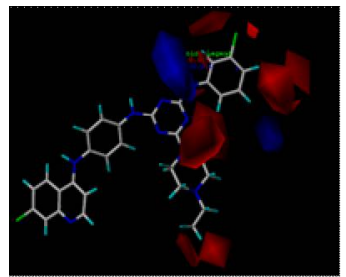

A

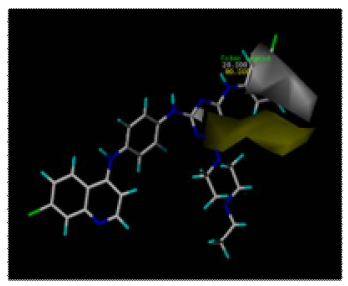

C

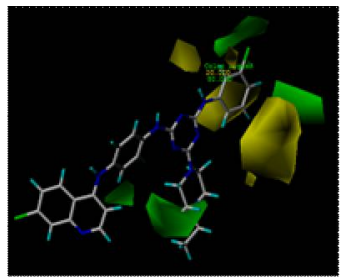

B

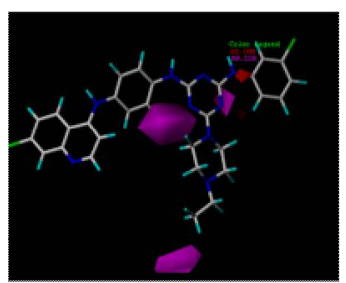

D
Figure 3: Contour maps overlapped with molecule 35: A. Electrostatic contour map for selected CoMFA model; B. Steric contour map for selected CoMFA model; C. Hydrophobic contour map for model CoMSIA EHA; D. Hydrogen bond acceptor contour map for model CoMSIA_EHA

\section{Molecule design, applicability domain analysis, and activity prediction}

Based on the findings from QSAR studies, We successfully acquired robust and highly predictive models and summarized the overall requirements of substituent properties for rational molecular design: 1 . arylamine group on triazines should be reserved and any substitution on aromatic ring should be executed on position three or position four; 2 . It is beneficial to keep the piperazine group, or potential benefits may be realized if piperazine is replaced with any bulky ring group with hydrogen bond forming characteristics; 3 . The aliphatic chain portion on the piperazine should be reserved and addition of a negative electrostatic favored chain terminal with hydrogen bond forming ability may achieve drug development objectives.

Development of the models and molecular design requirements were followed by examination of 89 molecules to discover potential anti-malaria agents. Each designed molecule was processed using the same structural optimization approach used for the dataset compounds. Optimum prediction space analysis (Discovery Studio software) was performed to build the applicability domain for the created models. Thirty-seven of all the designed compounds were evaluated as being unreliable for use in the created models. The remaining molecules were aligned with the molecule 35 reference for activity prediction, and compounds 
wm $\times 09$ and $w m \times 25$ were predicted to have better activity than molecule 35 (Table 9). We then examined these two compounds. A superimposed mapping analysis was performed to reveal the correlations for the contour maps and the designed molecules and then certify if the two compounds were rationally predicted. As presented in Figure $4 \mathrm{~A}$ and Figure 4B, the modified cyano group of compound wm $\times 09$ orients to the green and red mixed region. Its bulky and electron-withdrawing properties met the requirements. The results presented in Figure 4E and Figure 4F indicate that substituent group of the amide on phenylamino of compound wm $\times 25$ was assigned to an interlaced region with red and green modules. Electron-withdrawing oxygen atoms directly orients to a negative electrostatic favored region, indicating that a rational placement for the amide group was position four on the aromatic ring. Modification of the amide group at the aliphatic chain terminal satisfactorily met the negative electrostatic favored and hydrogen bond forming requirements (red and purple modules) (Figure 4 $B, D, F$ and $H$ ).

Table 9: Molecular structures and predicted activities of the screened compounds of wmx09 and wmx25

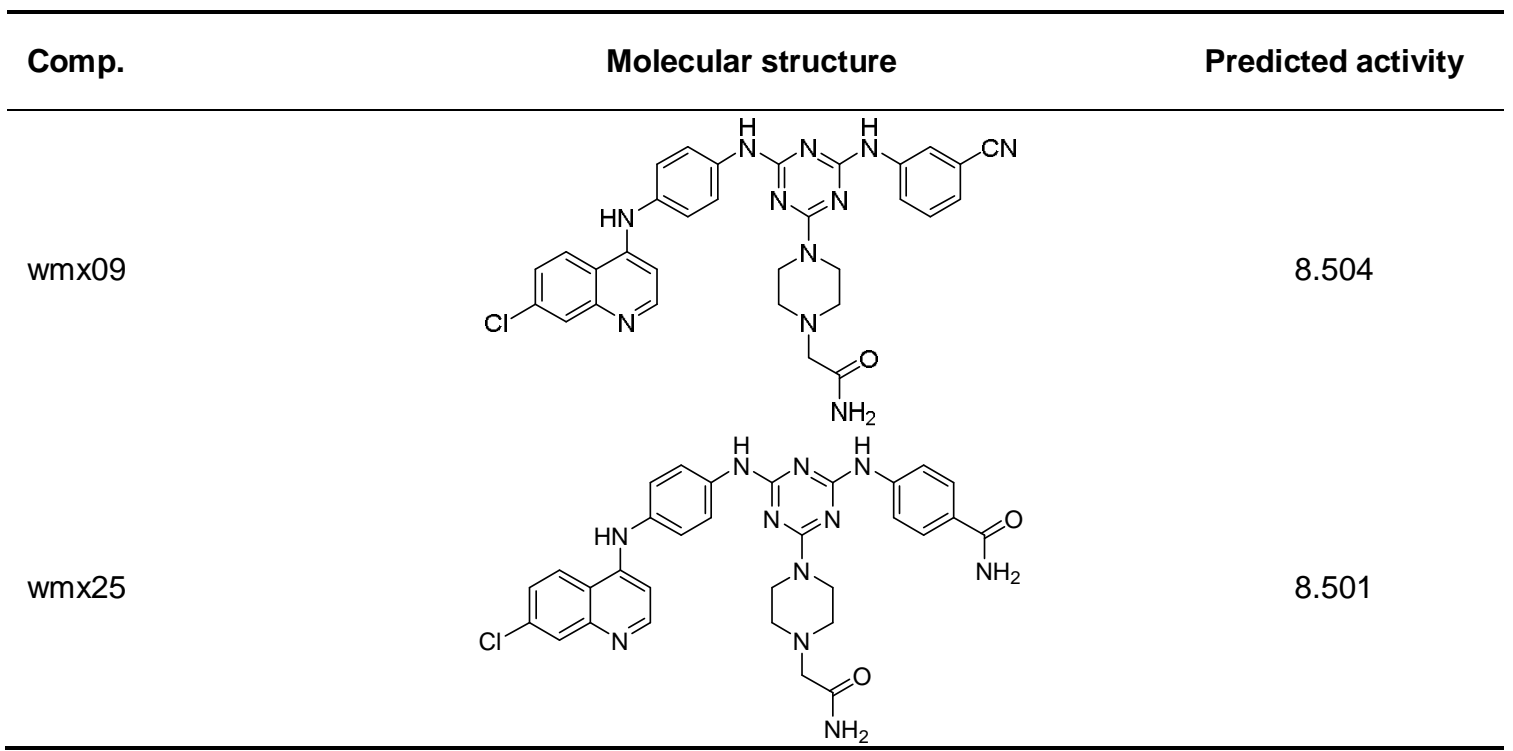

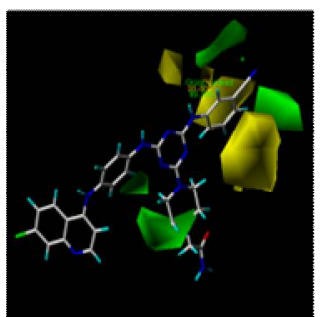

A

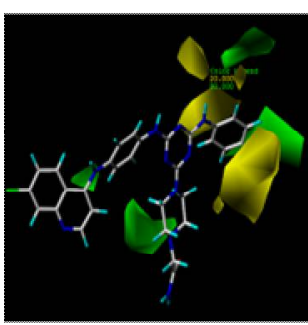

E

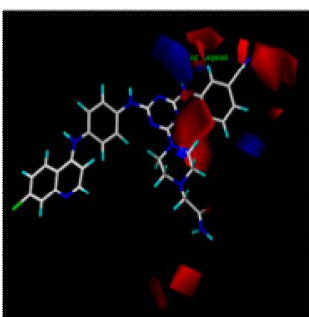

B

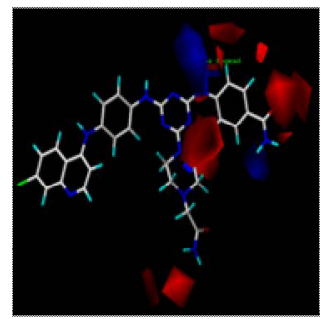

F

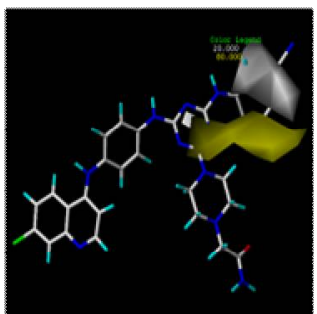

C

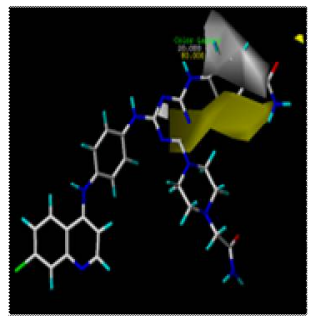

G

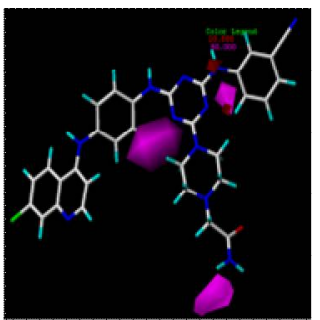

D

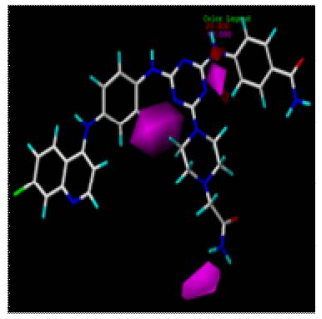

H

Figure 4: A - D. Contour maps overlapped with compound wmx09: A. Electrostatic contour map of selected CoMFA model; B. Steric contour map of selected CoMFA model; C. Hydrophobic contour map of model CoMSIA_EHA; D. Hydrogen bond acceptor contour map of model CoMSIA_EHA; E - F. Contour maps overlapped with compound wmx25: A. Electrostatic contour map of selected CoMFA model; B. Steric contour map of selected CoMFA model; C. Hydrophobic contour map of model CoMSIA_EHA; D. Hydrogen bond acceptor contour map of model CoMSIA_EHA 
Table 10: ADMET prediction outcomes for both designed compounds

\begin{tabular}{cccccc}
\hline \multirow{2}{*}{ Comp. } & \multicolumn{5}{c}{ ADMET descriptor } \\
\cline { 2 - 6 } & $\begin{array}{c}\text { Intestinal } \\
\text { absorption level }\end{array}$ & $\begin{array}{c}\text { Aqueous solubility } \\
\text { level }\end{array}$ & $\begin{array}{c}\text { BBB penetration } \\
\text { level }\end{array}$ & $\begin{array}{c}\text { CYP2D6 } \\
\text { binding }\end{array}$ & Hepatotoxicity \\
\hline$w m \times 09$ & 2 & 2 & 3 & True & True \\
$w m \times 25$ & 1 & 2 & 3 & False & True \\
\hline
\end{tabular}

\section{ADMET prediction}

ADMET investigation was performed for further molecular verification using "ADMET Descriptors" in the Discovery Studio software. The prediction outcomes for compound wmx09 and wm×25 are presented in Table 10. Both compounds were predicted to have acceptable solubility at level of $2(-6.0<\log (\mathrm{Sw})<-4.1)$ and weak blood-brain barrier penetration abilities. Compound wm×25 was predicted to have relatively better outcomes of CYP2D6 inhibition and intestinal absorption. However, both compounds were evaluated to have potential hepatotoxicity, so additional changes should be made to reduce this toxicity. The "toxicity prediction" function in the Discovery Studio software was used for the molecular toxicity investigation. The results indicated that both compounds had acceptable toxicity characteristics. Compound wmx09 was predicted to have female rodent carcinogenicity based on National Toxicology Program criteria. Compound wm $\times 25$ was predicted to have skin sensitization characteristics. In general, acceptable ADMET investigation results were acquired for both designed compounds. However, more work is necessary to improve their drug-like characteristics before they can qualify as lead compounds for further study.

\section{DISCUSSION}

The present study was designed and performed to screen for potential anti-malaria agents based on 4-anilinoquinoline analogs. After a systematic model selection approach, CoMFA and CoMSIA_EHA QSAR models built using molecular partition approach 02 were chosen depending on their relatively optimal statistical values. Carefully analysis of created contour maps provided informative clues leading to overall requirements for a molecular design. Subsequent activity prediction based on the chosen models assisted us to discover two

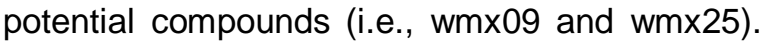
The results of the superimposed mapping analysis reinforced the prediction outcomes by displaying several favorable interactions between the designed compounds and different contour regions. The evaluation using the AMDET approach also found that compound wmx09 and wm $\times 25$ with acceptable results.

\section{CONCLUSION}

Based on a series of 4-aminoquinolinyl analogs, we built robust QSAR predictive models. The results of subsequent molecular screening studies indicated that compounds wmx09 and wmx25 have high potential as anti-malaria agents. Further research on these two compounds would have considerable scientific value.

\section{DECLARATIONS}

\section{Acknowledgement}

This work was funded by the National Natural Science Foundation of China (no. 81803732).

\section{Conflict of interest}

No conflict of interest is associated with this work.

\section{Contribution of authors}

The authors declare that this work was done by the authors named in this article and all liabilities pertaining to claims relating to the content of this article will be borne by them. Authors declare this manuscript submitted has not been previously published, nor is it before another journal for consideration. Bo $\mathrm{Wu}$ and Jiao-long Wang contributed to the work equally and should be regarded as co-first authors. Chong-Bo Zhao and Chun-Jie Wu conceived and designed the study. Bo Wu, Jiao-Long Wang and Liang Li collected and analyzed the data. Bo Wu and Jiao-Long Wang wrote the manuscript. Bo Wu and JiaoLong Wang did the detail experiments. All authors have read and approved the manuscript for publication.

\section{Open Access}

This is an Open Access article that uses a funding model which does not charge readers or their institutions for access and distributed under the terms of the Creative Commons Attribution License (http://creativecommons.org/licenses/by/ 4.0) and the Budapest Open Access Initiative 
(http://www.budapestopenaccessinitiative.org/rea d), which permit unrestricted use, distribution, and reproduction in any medium, provided the original work is properly credited.

\section{REFERENCES}

1. World Health Organization. World malaria report 2018. Geneva: WHO Press 2018: 1-7.

2. Frevert U. Sneaking in through the back entrance: the biology of malaria liver stages. Trend Parasitol 2004; 20 : 417-424.

3. Narasimhan V, Attaran A. Roll Back Malaria? The scarcity of international aid for malaria control. Malar $J$ 2003; 2(1): 8.

4. Ridley RG. Medical need, scientific opportunity and the drive for antimalarial drugs. Nature 2002; 415(6872): 686-693.

5. Sparatore A, Basilico N, Parapini S, Romeo S, Novelli F, Sparatore F, Taramelli $D$. 4-Aminoquinoline quinolizidinyl- and quinolizidinylalkyl-derivatives with antimalarial activity. Bioorg Med Chem 2005; 13(18): 5338-5345.

6. Iwaniuk $D P$, Whetmore $E D$, Rosa $N$, Ekoue-Kovi $K$, Alumasa J, de Dios AC, Poepe PD, Wolf C. Synthesis and antimalarial activity of new chloroquine analogues carrying a multifunctional linear side chain. Bioorg Med Chem 2009; 17(18): 6560-6566.

7. Roy PP, Paul S, Mitra I, Roy K. On two novel parameters for validation of predictive QSAR models. Molecules 2009; 14(5): 1660-1701.

8. Chitre TS, Asgaonkar KD, Miniyar PB, Dharme AB, Arkile $M A$, Yeware A, Khedkar VM, Jha PC. Synthesis and docking studies of pyrazine-thiazolidinone hybrid scaffold targeting dormant tuberculosis. Bioorg Med Chem Lett 2016; 26(9): 2224-2228.

9. Gnerre $C$, Catto $M$, Leonetti $F$, Weber $P$, Carrupt $P A$, Altomare C, Carotti A, Testa B. Inhibition of Monoamine Oxidases by Functionalized Coumarin Derivatives: Biological Activities, QSARs, and 3D-QSARs. J Med Chem 2000; 43(25): 4747-4758.

10. Assefa H, Buolamwini JK. CoMFA and CoMSIA $3 D$ QSAR and Docking Studies on ConformationallyRestrained Cinnamoyl HIV-1 Integrase Inhibitors: Exploration of a Binding Mode at the Active Site. J Med Chem 2002; 45(4): 841-852.

11. Kumar A, Srivastava K, Kumar SR, Siddiqi MI, Puri SK, Sexana JK, Chauhan PM. 4-Anilinoquinoline triazines: $A$ novel class of hybrid antimalarial agents. Eur $J$ Med Chem 2011; 46(2): 675-690.

12. Sharma M, Chauhan K, Chauhan SS, Kumar A, Singh SV, Saxena JK, Agarwal P, Srivastava K, Kumar SR, Puri SK, Shah P, Siddiqi MI, Chauhan PM. Synthesis of hybrid 4-anilinoquinoline triazines as potent antimalarial agents, their in silico modeling and bioevaluation as Plasmodium falciparum transketolase and $\beta$-hematin inhibitors. MedChemComm 2012; 3(1): 71-79.
13. Sharma $M$, Chauhan $K$, Srivastava RK, Singh SV, Srivastava K, Saxena JK, Puri SK, Chauhan PM. Design and synthesis of a new class of 4-aminoquinolinyl- and 9-anilinoacridinyl Schiff base hydrazones as potent antimalarial agents. Chem Biol Drug Des 2014; 84(2): 175-181.

14. Elgamacy MA, Shalaby RA, Elkodsh AT, kamel $A F$, Elsayed MSA, Abou-El-Ella D. New inhibitors of VEGFR-2 targeting the extracellular domain dimerization process. Bioinformation 2011; 7(2): 52-58.

15. Sprous DG. Fingerprint-based clustering applied to define a QSAR model use radius. J Mol Graphics Modell 2008; 27(2): 225-232.

16. Wang JL, Li L, Hu MB, Wu B, Fan WX, Peng W, Wei DN, Wu CJ. In silico drug design of inhibitor of nuclear factor kappa $B$ kinase subunit beta inhibitors from 2acylamino-3-aminothienopyridines based on quantitative structure-activity relationships and molecular docking. Comput Biol Chem 2019; 78: 297-305.

17. Li W, Si H, Li Y, Ge CZ, Song FC, Ma XT, Duan YB, Zhai $H L$. 3D-QSAR and molecular docking studies on designing inhibitors of the Hepatitis C Virus NS5B polymerase. J Mol Struct 2016; 1117: 227-239.

18. Hao CZ, Xia SW, Wang $H$, Xue J, Yu LM. Using $3 D-$ QSAR and molecular docking insight into inhibitors binding with complex-associated kinases CDK8. J Mol Struct 2018; 1174: 498-511.

19. Nagarajan SK, Babu S, Sohn H, Devaraju P, Madhavan T. 3D-QSAR studies on indole and 7-azoindole derivatives as ROCK-2 inhibitors: An integrative computational approach. Comput Biol Chem 2017; 71: 104-116.

20. Buolamwini JK, Assefa H. CoMFA and CoMSIA 3D QSAR and docking studies on conformationallyrestrained cinnamoyl HIV-1 integrase inhibitors: exploration of a binding mode at the active site. $J$ Med Chem 2002; 45(4): 841-852.

21. Gramatica P. Principles of QSAR models validation: internal and external. QSAR Comb Sci 2007; 26(5): 694701

22. Oprea TI, Waller CL, Marshall GR. Three-dimensional quantitative structure-activity relationship of human immunodeficiency virus (I) protease inhibitors. 2. Predictive power using limited exploration of alternate binding modes. J Med Chem 1994; 37(14): 2206-2215.

23. Sahigara F, Mansouri K, Ballabio D, Mauri A, Consonni $V$, Todeschini R. Comparison of different approaches to define the applicability domain of QSAR models. Molecules 2012; 17(5): 4791-4810.

24. Cheng A, Merz JK. Prediction of aqueous solubility of a diverse set of compounds using quantitative structureproperty relationships. J Med Chem 2003; 46(17): 35723580.

25. Cheng A, Dixon SL. In silico models for the prediction of dose-dependent human hepatotoxicity. J Comput-Aided Mol Des 2003; 17(12): 811-823. 